\title{
Development of a Loop-Mediated Isothermal Amplification Assay for Rapid Detection of BK Virus
}

\author{
Bipin Raj Bista, ${ }^{1}$ Chandra Ishwad, ${ }^{1}$ Robert M. Wadowsky, ${ }^{2}$ Pradip Manna, ${ }^{3}$ Parmjeet Singh Randhawa, ${ }^{4}$ \\ Gaurav Gupta, ${ }^{1}$ Meena Adhikari, ${ }^{5}$ Rakhi Tyagi, ${ }^{5}$ Gina Gasper, ${ }^{1}$ and Abhay Vats ${ }^{1 *}$ \\ Department of Pediatrics, Children's Hospital of Pittsburgh, 3705 Fifth Avenue, Pittsburgh, Pennsylvania 15213'; Department of Pathology, \\ Children's Hospital of Pittsburgh, 3705 Fifth Avenue, Pittsburgh, Pennsylvania 15213²; Department of Molecular Diagnostics, \\ ViraCor Laboratories, 1210 NE Windsor Drive, Lee's Summit, Missouri 64086 ${ }^{3}$; Department of Pathology, University of \\ Pittsburgh Medical Center, 200 Lothrop Street, Pittsburgh, Pennsylvania 15213 ; and Department of \\ Molecular Virology, Atharva BioSciences, New Delhi 110029, India 5
}

Received 16 May 2006/Returned for modification 6 July 2006/Accepted 14 December 2006

\begin{abstract}
Loop-mediated isothermal amplification (LAMP) is a novel method for rapid amplification of DNA. Its advantages include rapidity and minimal equipment requirement. The LAMP assay was developed for BK virus (BKV), which is a leading cause of morbidity in renal transplant recipients. The characteristics of the assay, including its specificity and sensitivity, were evaluated. BKV LAMP was performed using various incubation times with a variety of specimens, including unprocessed urine and plasma samples. A ladder pattern on gel electrophoresis, typical of successful LAMP reactions, was observed specifically only for BKV and not for other viruses. The sensitivity of the assay with $1 \mathrm{~h}$ of incubation was 100 copies/tube of a cloned BKV fragment. Additionally, a positive reaction was visually ascertained by a simple color reaction using SYBR green dye. BKV LAMP was also successful for urine and plasma specimens without the need for DNA extraction. Due to its simplicity and specificity, the LAMP assay can potentially be developed for "point of care" screening of BKV.
\end{abstract}

BK virus (BKV) can cause renal allograft nephropathy (BKVAN), which is now recognized as one of the major manifestations of the various syndromes associated with polyomaviruses (4-6). Currently, 1 to $10 \%$ of renal transplant recipients are diagnosed with BKVAN, which can lead to graft loss in 20 to $80 \%$ of patients $(3,7,13,19)$. Recent studies have also suggested a viruria prevalence of approximately $30 \%$ and a viremia prevalence of 7 to $10 \%$ in patients with renal transplants $(2,4-6,16,19)$. Over the last several years, we developed and brought into clinical practice a quantitative real-time (TaqMan) PCR assay for BKV $(16,19)$. We have also shown that screening by monitoring of viral load in urine can predict patients at risk for development of BKVAN (16). The cornerstones of BKVAN management are an early diagnosis followed by a reduction in immunosuppression with or without antiviral therapy $(4-7,19)$. Thus, different PCR techniques have now become a valuable tool for screening and monitoring for active BKV infection $(7,16,19)$. However, PCR assays require considerable operator skill and expensive equipment. Rapid diagnostic tests that can be performed in the outpatient clinic without the need for complex procedures (like DNA extraction) or expensive equipment can potentially improve our capability to screen for this important transplant complication.

Recently, a novel, specific, and rapid technique for amplification of DNA under isothermal conditions was reported (14). The technique, termed loop-mediated isothermal amplification (LAMP), requires a set of conditions and primers different

\footnotetext{
* Corresponding author. Mailing address: Department of Pediatrics, Children's Hospital of Pittsburgh, 3705 Fifth Avenue, Pittsburgh, PA 15213. Phone: (412) 692-5182. Fax: (412) 692-7443. E-mail: abhay.vats @ chp.edu.

${ }^{\nabla}$ Published ahead of print on 21 February 2007.
}

from those used for PCRs $(8,12,14,15)$. LAMP assays have now been reported for several pathogens $(8,9,12,14,15,18$, $20)$. The reaction typically occurs over 30 to $60 \mathrm{~min}$ under isothermal conditions with temperatures ranging from 60 to $65^{\circ} \mathrm{C}$ and can be conducted with a simple heating block. Thus, the thermal-cycling needs of a PCR are avoided. In this study, we developed and characterized LAMP primers and amplification protocols for a BKV assay (U.S. patents pending). We report the details of this new technique and the assay characteristics.

\section{MATERIALS AND METHODS}

Patient samples. Urine and blood samples obtained from renal transplantation patients and stored at $-80^{\circ} \mathrm{C}$ were utilized for development and testing of BKV LAMP assays and for comparison with PCR assays. The patients signed an informed consent, and the studies were approved by the Internal Review Board of the University of Pittsburgh Medical Center and the Children's Hospital of Pittsburgh (CHP). Anonymous samples were obtained from ViraCor Laboratories and the microbiology laboratories of CHP for various viruses.

Viral DNA extraction. Viral DNA was extracted from blood samples by using a QIAmp DNA mini kit (QIAGEN Inc., CA). A QIAmp viral RNA kit was used to extract viral DNA from urine samples, as we have found that this method provides a better yield for urinary viral DNA extraction than the use of a DNA kit $(16,17)$. The DNA was finally eluted in a $60-\mu l$ volume of elution buffer and stored at $-20^{\circ} \mathrm{C}$ until further use.

LAMP reaction. The LAMP reaction was conducted, following methods described by Notomi et al. and Nagamine et al. with minor modifications $(12,14)$ Primers specific for BKV were designed and synthesized (Integrated DNA Technologies, IA). The six primers consisted of one pair each of forward and reverse outer primers (BKVTF3 and BKVTB3), forward and reverse inner primers (BKVTFIP and BKVTBIP), and forward and reverse loop primers (BKVTloopF and BKVTloopB). The LAMP reaction mixtures (final volume, $25 \mu \mathrm{l}$ ) contained these three primer pairs in a 1:8:4 ratio, i.e., $0.2 \mu \mathrm{M}$ each of the outer primers, $1.6 \mu \mathrm{M}$ each of the inner primers, and $0.8 \mu \mathrm{M}$ each of the loop primers. The other components of the reaction mixture were $2.5 \mu \mathrm{l}$ of $10 \times$ Bst DNA polymerase reaction buffer (New England Biolabs, MA), $1 \mu \mathrm{l}$ of $8 \mathrm{U} / \mu \mathrm{l}$ Bst DNA polymerase (New England Biolabs Inc., MA), $2 \mathrm{mM}$ of $\mathrm{MgSO}_{4}(2 \mu \mathrm{l}), 5 \mu \mathrm{l}$ of Betaine (Sigma-Aldrich, MO), and $5 \mu \mathrm{l}$ of a target sample. The Bst DNA polymerase reaction buffer $(1 \times)$ contained $20 \mathrm{mM}$ Tris- $\mathrm{HCl}\left(\mathrm{pH} 8.8,25^{\circ} \mathrm{C}\right), 10$ 
TABLE 1. LAMP primers

\begin{tabular}{|c|c|c|}
\hline Primer used for BKV LAMP & Sequence & No. of bases \\
\hline \multicolumn{3}{|l|}{ Outer forward and backward } \\
\hline BKVTF3 & GGACCCACCATTGCAGAGTTT (outer forward primer) & 21 \\
\hline BKVTB3 & TCTTTGCCCAGATACCCTGT (outer primer) & 20 \\
\hline \multicolumn{3}{|l|}{ Inner forward and backward ${ }^{a}$} \\
\hline BKVTBIP $(\mathrm{B} 1+\mathrm{B} 2 \mathrm{c})$ & TTTAAATGYCTTAATCTWAGCTGAGGAWTGGCCTATTTGTTCCA & 44 \\
\hline BKVTFIP (F1c + F2) & TTTTAAGAAAAGAGCCCTTGGTTAGGTCTAAGCCAAACCACTGT & 44 \\
\hline \multicolumn{3}{|l|}{ Loop forward and backward } \\
\hline BKVTLoop F & GATTGCTACTGCATTGACTGCT & 22 \\
\hline BKVTLoop B & ATGCAAGGGCAGTGCAC & 17 \\
\hline
\end{tabular}

${ }^{a}$ The F1c and B2c sequences are complementary and reverse to the F1 and B2 regions shown in Fig. 1 and are shown as underlined. The BKVTBIP primer had two degenerate bases in three positions $(\mathrm{W}=\mathrm{A} / \mathrm{T}$ and $\mathrm{Y}=\mathrm{C} / \mathrm{T})$.

$\mathrm{mM} \mathrm{KCl}, 10 \mathrm{mM}\left(\mathrm{NH}_{2}\right) \mathrm{SO}_{4}, 2 \mathrm{mM} \mathrm{MgSO}_{4}$, and $0.1 \%$ Triton X-100. The reaction mixtures were incubated at a temperature of $63^{\circ} \mathrm{C}$ for various periods of time, ranging from 30 to $120 \mathrm{~min}$

LAMP product detection. As part of the assay development, the LAMP products were initially detected by several methods, including electrophoresis using agarose gel $(1.2 \%)$ with UV light transillumination and photography using the KODAK EDAS gel documentation system (Eastman Kodak Company, Rochester, NY). A portable UV transilluminator was also used (FastGel system; Cambrex BioSciences Inc., Rockland, ME) for LAMP product visualization. In addition, the products were detected visually by a color change after addition of SYBR green I dye to tubes containing LAMP products. Ten microliters of SYBR green I dye $(100 \times)$ (Cambrex BioSciences Inc., Rockland, ME) was added to each tube containing LAMP products. The LAMP products were also detected spectrophotometrically (SmartSpec 3000; Bio-Rad Laboratories, CA) at wavelengths of 494 to $521 \mathrm{~nm}$. After the correlation was established between various detection methods, the SYBR green dye method was used for evaluation of clinical samples.

Amplification targets and LAMP equipment. BKV DNA was used to standardize the LAMP reaction and to determine the specificity of the assay. DNA extracted from cytomegalovirus (CMV), Epstein-Barr virus (EBV), human herpesvirus 6 (HHV-6), adenovirus (Ad), herpes simplex virus 1 and 2 (HSV-1 and HSV-2), varicella-zoster virus (VZV), and other polyomaviruses (simian virus 40 [SV40] and JC virus [JCV]) was used to determine the specificity of the BKV LAMP reaction. CMV-, EBV-, HHV-6-, Ad-, HSV-1-, HSV-2-, VZV-, and SV-40-positive patient blood or urine samples were obtained from the microbiology laboratories of CHP and ViraCor. JCV was obtained from the American Type Culture Collection (ATCC number 45027). A plasmid containing a cloned target sequence of BKV (pBKVT3) was used to determine the sensitivity of the reaction. The negative controls used for various reactions included no-template controls and no-enzyme controls. The LAMP reaction was primarily carried out with a heating block (LAB-LINE; Barnstead International, Dubuque, IA). The reaction was also performed with conventional PCR (MyCycler; Bio-Rad Laboratories, CA) and real-time PCR (Mini-Opticon; Bio-Rad Laboratories, CA) machines.

Cloning of BKV DNA. To determine the sensitivity of the BKV LAMP assay, we generated and quantitated a plasmid containing the target BKV sequence (pBKVT3). Briefly, a 217-bp target DNA sequence spanning the region from nucleotide position 4676 to nucleotide 4893 of the BKV genome was amplified by PCR using the same outer primers as those used in the LAMP reaction (BKVTF3 and BKVTB3). The amplified product was then cloned into a TOPO cloning vector, using a TA cloning kit according to the manufacturer's instructions (Invitrogen, CA). The vector was used to transform XL1-Blue competent Escherichia coli cells (Stratagene, CA). The transformed cells were incubated overnight and the colonies with the insert (using blue-white distinction) further grown. The cloned insert was isolated from the cells by use of a Fast Plasmid min kit (Eppendorf, NY). The presence of the positive clone was tested by digestion of the plasmid DNA by EcoRI, followed by gel electrophoresis and sequencing. The pBKVT3 clone was quantitated using UV spectrophotometry at $260 \mathrm{~nm}$ (SmartSpec 3000; Bio-Rad Laboratories, CA). A series of $10 \times$ dilutions, spanning 10 to 10E7 copies/tube of the clone, was used to test the sensitivity of the LAMP reactions.

Real-time PCR. The real-time PCR technique was used to quantify the BKV DNA as previously described $(16,19)$ and to compare the sensitivities of the
LAMP and PCR assays. PCRs were performed using the Takara PCR protocol (TakaraMirus Bio Inc., WI). The reaction mixtures $(20 \mu \mathrm{l})$ contained $10 \mu \mathrm{l}$ of Takara master mix, $0.67 \mu \mathrm{l}$ each of $10 \mathrm{mM}$ forward and reverse primers, $0.67 \mu \mathrm{l}$ of $10 \mathrm{mM}$ probe, and $5 \mu \mathrm{l}$ of the sample. Thermal cycling and quantitation were performed using a Bio-Rad Mini-Opticon machine (Bio-Rad Laboratories, CA). The reaction mixture was cycled as follows: $95^{\circ} \mathrm{C}$ for 30 seconds and 40 cycles of two-step PCR, with each cycle consisting of $95^{\circ} \mathrm{C}$ for $1 \mathrm{~s}$, followed by $60^{\circ} \mathrm{C}$ for $5 \mathrm{~s}$, with plate reading after each cycle. The primers and probe used for the real-time PCR are located in the cloned plasmid (pBKVT3), and the sequences were as follows: forward, GGACCCACCATTGCAGAGTTT; reverse, AGAGCCCT TGGTTTGGATAGATT; probe, 6-FAM (6-carboxyfluorescein)-5'-AAGC CAAACCACTGTGTGAAGCAGTCAAT 3'-TAMRA (6-carboxytetramethylrhodamine). The real-time measurement of BK viral load in clinical specimens also utilized an internal control. Each specimen was spiked with a universal internal control (UIC) prior to DNA extraction. The UIC contains a partial gene sequence for green fluorescence protein that is absent in viral-pathogen and mammalian genomes. To assess for PCR inhibition, a separate real-time PCR assay was performed with UIC-specific primers and probe. Real-time PCR inhibition was defined by the absence of UIC target amplification.

\section{RESULTS}

Assay design. The LAMP primers were selected from a conserved region of the BKV genome as determined by wholegenome sequencing of several viral strains obtained from different BKV-positive patient samples and are shown in Table 1. The locations and sequences of the different primers are shown in Fig. 1. All primers were designed by manual inspection of

\section{AAAGCTTTAGATCTCTGAAGGGAGTTTCTCCAATTATTTGGACCCACCATTGCAGAGTTT}

E3 ${ }_{4697}$ CTTCAGTTAGGTCTAAGCCAAACCACTGTGTGAAGCAGTCAATGCAGTAGCAATCTATCC

$$
\text { F2 } \quad+100 \mathrm{p} \mathrm{F}
$$

4757 AAACCAAGGGCTCTTTTCTTAAAAATTTTCTATTTAAATGCCTTAATCTAAGCTGACATA

B1

\section{GCATGCAAGGGCAGTGCACAGAAGGCTTTTTGGAACAAATAGGCCATTCCTTGCAGTACA}

loop $B \rightarrow$

B2

\section{GGGTATCTGGGCAAAGAGGAAAATCAGCACAAACCTCTGAGCTACTCCAGGTTCCA}

\section{B3}

FIG. 1. Segment of large T-antigen gene sequence (nucleotide positions 4676 to 4893 ) showing the locations and orientations of various primers used in the BKV LAMP assay. 
the sequence data. Briefly, the outer primers (F3 [BKVTF3] and B3 [BKVTB3]) were located outside two inner primers (labeled F2 [BKVTF2] and B2 [BKVTB2]) in the large-Tantigen gene region of the BKV genome. The forward internal primer (BKVTFIP) comprises a fusion primer with the complementary F1 sequence (F1c) and the direct F2 sequences (14). Similarly, the reverse internal primer (BKVTBIP) comprises the direct B1 sequence and the complementary B2 sequence (B2c) (Table 1). To increase the amplification efficiency, two loop primers (forward [BKVTloopF] and reverse [BKVTloopB]) were also designed. The loop primers significantly improved the performance of the assay and led to a considerable reduction in the time required for amplification of the target. The LAMP reaction was carried out at a constant temperature of $63^{\circ} \mathrm{C}$ and generally was positive after various periods of time (30 to $120 \mathrm{~min}$ ) with the addition of loop primers to the reaction mixture. Without the use of loop primers, the same reactions required periods 4 to 5 times longer (i.e., a 30-min reaction required over 2 or $3 \mathrm{~h}$ ). Also, several samples with lower viral loads could not be amplified at all without the addition of loop primers.

Assay specificity and sensitivity. A positive BKV LAMP reaction typically required incubation for $60 \mathrm{~min}$ and revealed a ladder pattern with many bands of different sizes on agarose gel electrophoresis (Fig. 2A, BKV lane). The specificity of the BKV LAMP assay was evaluated by testing the assay on several DNA viruses that can be seen in transplant patients (i.e., EBV, CMV, HHV-6, Ad, HSV-1, HSV-2, and VZV) and the two related polyomaviruses (i.e., JCV and SV40). No ladder pattern was seen with the other (non-BK) viruses or for the negative control (Fig. 2A and B). The sensitivity of the LAMP reaction was determined using $10 \times$ serial dilutions of the pBKVT3 clone. The BKV LAMP sensitivity was found to be 100 copies per tube (Fig. 2C, lane 7) when an incubation period of $60 \mathrm{~min}$ was employed.

Detection of LAMP products by alternate methods. Besides gel electrophoresis, we also determined two additional methods, i.e., visual inspection and spectrophotometry, to detect a positive reaction. Upon addition of the SYBR green I dye to tubes after the LAMP reaction, the color changed to yellowish green in a positive reaction (Fig. 3A, tube 1) and remained reddish orange (the color of the unbound dye) in the negative reactions (Fig. 3A, tube 2). Although the intensities of the color changes appeared to be similar for a range of viral loads, the color changes occurred earlier in samples with higher loads. All the samples that were positive by gel electrophoresis were also positive by visual detection of color change (and vice versa). The visual detection of a positive reaction was further improved by using UV light from a conventional as well as a portable blue light transilluminator, which demonstrated a bright green fluorescence in positive reactions (Fig. 3B and C). Finally, the positive reaction was also detected by a spectrophotometer in a quantitative manner.

LAMP assay with clinical samples. Detection of BKV DNA by LAMP was done with 49 clinical blood and urine samples obtained from renal transplant patients. Real-time PCR assays for BKV were also done with the same samples for comparison. As there was a good correlation between various detection methods, positive-reaction detection with clinical samples was performed by visual observance of color change with SYBR
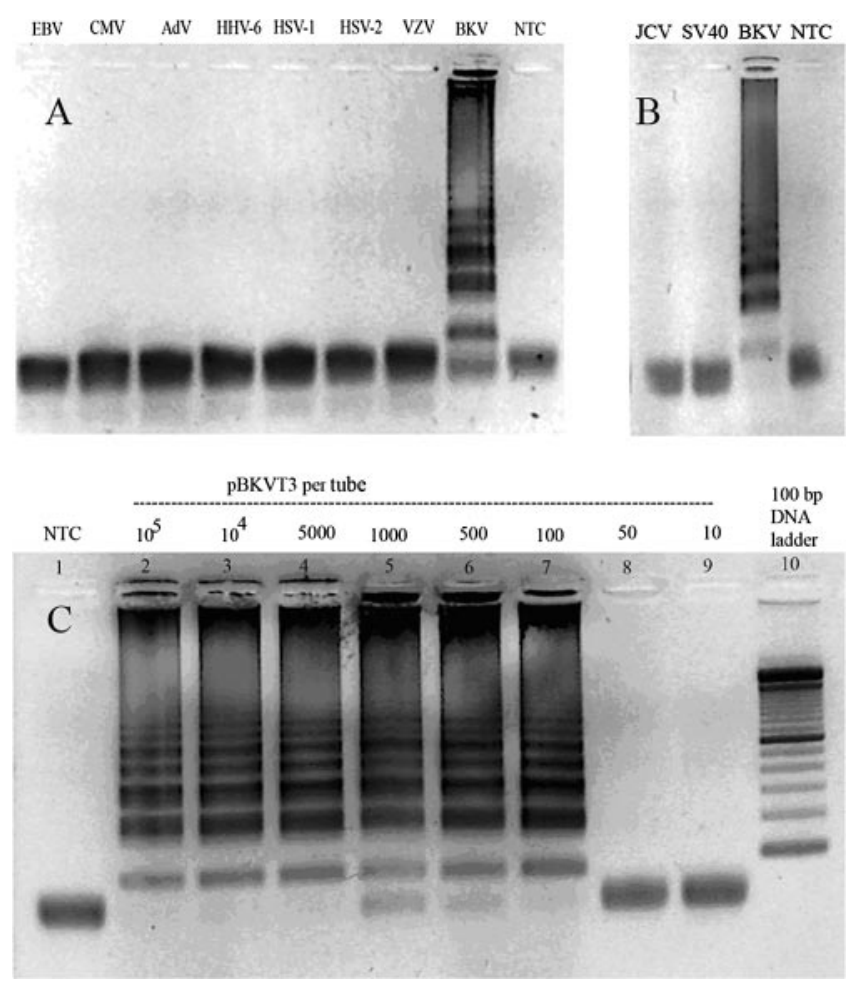

FIG. 2. Specificity and sensitivity of the BKV LAMP assay. (A) The specificity of the BKV LAMP assay is shown in the positive reaction, visible as a ladder-like pattern in lanes with BKV DNA only. A negative reaction was obtained when the target of amplification was other non-BKV DNA viruses, i.e., EBV, CMV, Ad, HHV-6, HSV-1, HSV-2, $\mathrm{VZV}$, or no-target control (NTC). (B) The LAMP reaction was negative for two related polyomaviruses, JCV and SV40, and amplified only BKV. (C) The BKV LAMP reaction was carried out with the serial dilutions of cloned BKV plasmid (pBKVT3), and the sensitivity of the reaction was determined to be 100 copies per tube.

green and UV transillumination. A summary of the results and the comparison between real-time PCR and LAMP is shown in Table 2. Plasma samples required approaches slightly different from those used for urine samples for LAMP to work successfully as described below.

Plasma samples. We analyzed 21 plasma samples from 18 different patients, and four of the plasma samples belonged to a single BKVAN patient (no. 18). These four samples were obtained over a period of 3 months and showed loads ranging from 2,264 to 333,200 copies/ml. LAMP was performed with extracted as well as unextracted plasma samples under two separate conditions, i.e., after heating at $99^{\circ} \mathrm{C}$ for $10 \mathrm{~min}$ and without heating (i.e., samples freshly obtained without any processing). The unprocessed and unextracted plasma samples did not give positive reactions with LAMP. However, the same samples were successfully amplified by LAMP after the plasma was heated. Approximately 15 to $20 \mu \mathrm{l}$ of supernatant was obtained after $50 \mu \mathrm{l}$ of plasma was heated, of which $5 \mu \mathrm{l}$ was used for each reaction (Fig. 4A). The LAMP assay was negative for samples that were negative by real-time PCR and also for three samples with viral loads of $<1,500$ copies $/ \mathrm{ml}$ (Table 2 ). The samples with viral loads of $<5,000$ copies $/ \mathrm{ml}$ required the LAMP reaction to be performed for a longer period (120 $\mathrm{min})$. 

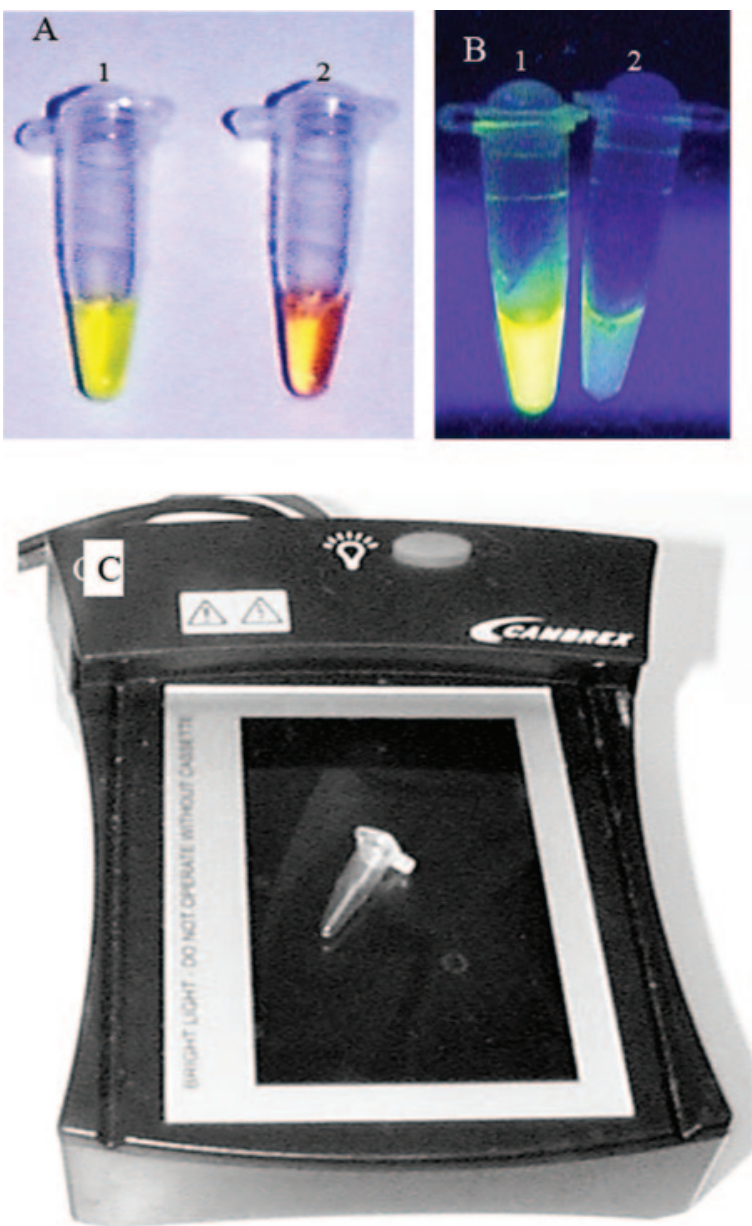

FIG. 3. Visual detection of LAMP products. (A) The BKV LAMP products were detected after addition of SYBR green I dye. The tube with a positive reaction (tube 1) shows a color change to yellowish green, which can be distinguished from the reddish orange color of a negative reaction. (B) The same positive sample, when visualized under UV transillumination, shows a bright green fluorescence in tube 1 (positive reaction) compared to what is observed for tube 2 (negative reaction). (C) A portable blue light illuminator that can be used in a clinic setting for transillumination of LAMP products. A $0.5-\mathrm{ml}$ PCR tube is placed on it to show the relative size of the transilluminator.

Urine samples. LAMP was performed with 28 urine samples from 27 patients, including 8 renal transplant recipients with asymptomatic viruria, 14 patients with biopsy-confirmed BKVAN, and 5 patients with no viruria. We initially analyzed the urine samples with and without (i.e., freshly obtained without any processing) DNA extraction from 10 patient samples (7 viruria and $3 \mathrm{BKVAN}$ ). Unlike for plasma, BKV LAMP performed successfully even when freshly voided (unprocessed) urine was added directly to the reaction mixture (Fig. 4B). The reactions were positive for unprocessed urine samples from four patients whose urine viral loads were $>50,000$ copies per $\mathrm{ml}$ (Fig. 4B). Interestingly, the assay was negative for two samples (Fig. 4B, lanes 1 and 3) with viral loads of approximately 60,000 and 40,000 copies/ml even after incubation for up to $2 \mathrm{~h}$. All 14 BKVAN patients were positive by the LAMP assay performed with unprocessed urine, including a patient with a low viral load of 30,500 copies per ml (Table 2). These results show that the sensitivity of LAMP is approximately 100,000 copies/ml for urinary samples, with variable performance at lower urinary viral loads.

\section{DISCUSSION}

In recent years, the detection of $\mathrm{BK}$ viremia and viruria by real-time PCR has become an important tool for identification of patients at risk for developing $\operatorname{BKVAN}(4,6,7,19)$. Despite

TABLE 2. Comparison of LAMP and real-time PCR results for clinical plasma and urine samples ${ }^{a}$

\begin{tabular}{|c|c|c|c|c|}
\hline Sample no. & $\begin{array}{l}\text { Patient } \\
\text { no. }\end{array}$ & Disease status & $\begin{array}{c}\text { BKV load } \\
\text { (real-time PCR) } \\
\text { (no. of copies/ml) }\end{array}$ & $\begin{array}{l}\text { LAMP } \\
\text { result }\end{array}$ \\
\hline \multicolumn{5}{|l|}{ Plasma } \\
\hline 1 & 1 & & No BKV & $\mathrm{Neg}$ \\
\hline 2 & 2 & & No BKV & Neg \\
\hline 3 & 3 & & No BKV & $\mathrm{Neg}$ \\
\hline 4 & 4 & & $8.00 \mathrm{E}+2$ & $\mathrm{Neg}$ \\
\hline 5 & 5 & & $1.40 \mathrm{E}+3$ & Neg \\
\hline 6 & 6 & & $1.43 \mathrm{E}+3$ & Neg \\
\hline 7 & 7 & & $5.00 \mathrm{E}+2$ & Pos \\
\hline 8 & 8 & & $2.50 \mathrm{E}+3$ & Pos \\
\hline 9 & 9 & & $3.35 E+3$ & Pos \\
\hline 10 & 10 & & $5.26 \mathrm{E}+3$ & Pos \\
\hline 11 & 11 & & $6.60 \mathrm{E}+3$ & Pos \\
\hline 12 & 12 & & $6.80 \mathrm{E}+3$ & Pos \\
\hline 13 & 13 & & $1.10 \mathrm{E}+4$ & Pos \\
\hline 14 & 14 & & $1.03 E+5$ & Pos \\
\hline 15 & 15 & & $1.42 \mathrm{E}+5$ & Pos \\
\hline 16 & 16 & & $1.40 \mathrm{E}+6$ & Pos \\
\hline 17 & 17 & & $2.60 \mathrm{E}+6$ & Pos \\
\hline 18 & 18 & & $2.26 \mathrm{E}+3$ & Pos \\
\hline 19 & 18 & & $5.05 \mathrm{E}+4$ & Pos \\
\hline 20 & 18 & & $1.57 \mathrm{E}+5$ & Pos \\
\hline 21 & 18 & & $3.33 \mathrm{E}+5$ & Pos \\
\hline
\end{tabular}

Urine

\begin{tabular}{lllll}
22 & 19 & Healthy control & No BKV & Neg \\
23 & 20 & Healthy control & No BKV & Neg \\
24 & 21 & Healthy control & No BKV & Neg \\
25 & 22 & Healthy control & No BKV & Neg \\
26 & 23 & Healthy control & No BKV & Neg \\
27 & 24 & Asympt viruria & $6.29 \mathrm{E}+3$ & Neg \\
28 & 25 & Asympt viruria & $2.09 \mathrm{E}+4$ & Neg \\
29 & 26 & Asympt viruria & $4.39 \mathrm{E}+4$ & Neg \\
30 & 27 & Asympt viruria & $5.84 \mathrm{E}+4$ & Neg \\
31 & 28 & Asympt viruria & $1.12 \mathrm{E}+5$ & Pos \\
32 & 29 & Asympt viruria & $3.98 \mathrm{E}+5$ & Pos \\
33 & 30 & BKVAN & $3.05 \mathrm{E}+4$ & Pos \\
34 & 31 & BKVAN & $5.22 \mathrm{E}+4$ & Pos \\
35 & 32 & Asympt viruria & $5.41 \mathrm{E}+4$ & Pos \\
36 & 33 & BKVAN & $5.37 \mathrm{E}+5$ & Pos \\
37 & 34 & BKVAN & $2.07 \mathrm{E}+7$ & Pos \\
38 & 35 & BKVAN & $1.05 \mathrm{E}+8$ & Pos \\
39 & 36 & Asympt viruria & $1.35 \mathrm{E}+8$ & Pos \\
40 & 37 & BKVAN & $4.39 \mathrm{E}+8$ & Pos \\
41 & 38 & BKVAN & $7.33 \mathrm{E}+8$ & Pos \\
42 & 39 & BKVAN & $1.05 \mathrm{E}+9$ & Pos \\
43 & 40 & BKVAN & $1.70 \mathrm{E}+9$ & Pos \\
44 & 41 & BKVAN & $3.40 \mathrm{E}+9$ & Pos \\
45 & 42 & BKVAN & $3.72 \mathrm{E}+9$ & Pos \\
46 & 43 & BKVAN & $9.98 \mathrm{E}+9$ & Pos \\
47 & 44 & BKVAN & $1.05 \mathrm{E}+10$ & Pos \\
48 & 45 & BKVAN & $1.65 \mathrm{E}+10$ & Pos \\
49 & 45 & BKVAN & $2.29 \mathrm{E}+10$ & Pos \\
\hline
\end{tabular}

${ }^{a}$ Asympt viruria, asymptomatic viruria; neg, negative; pos, positive. 

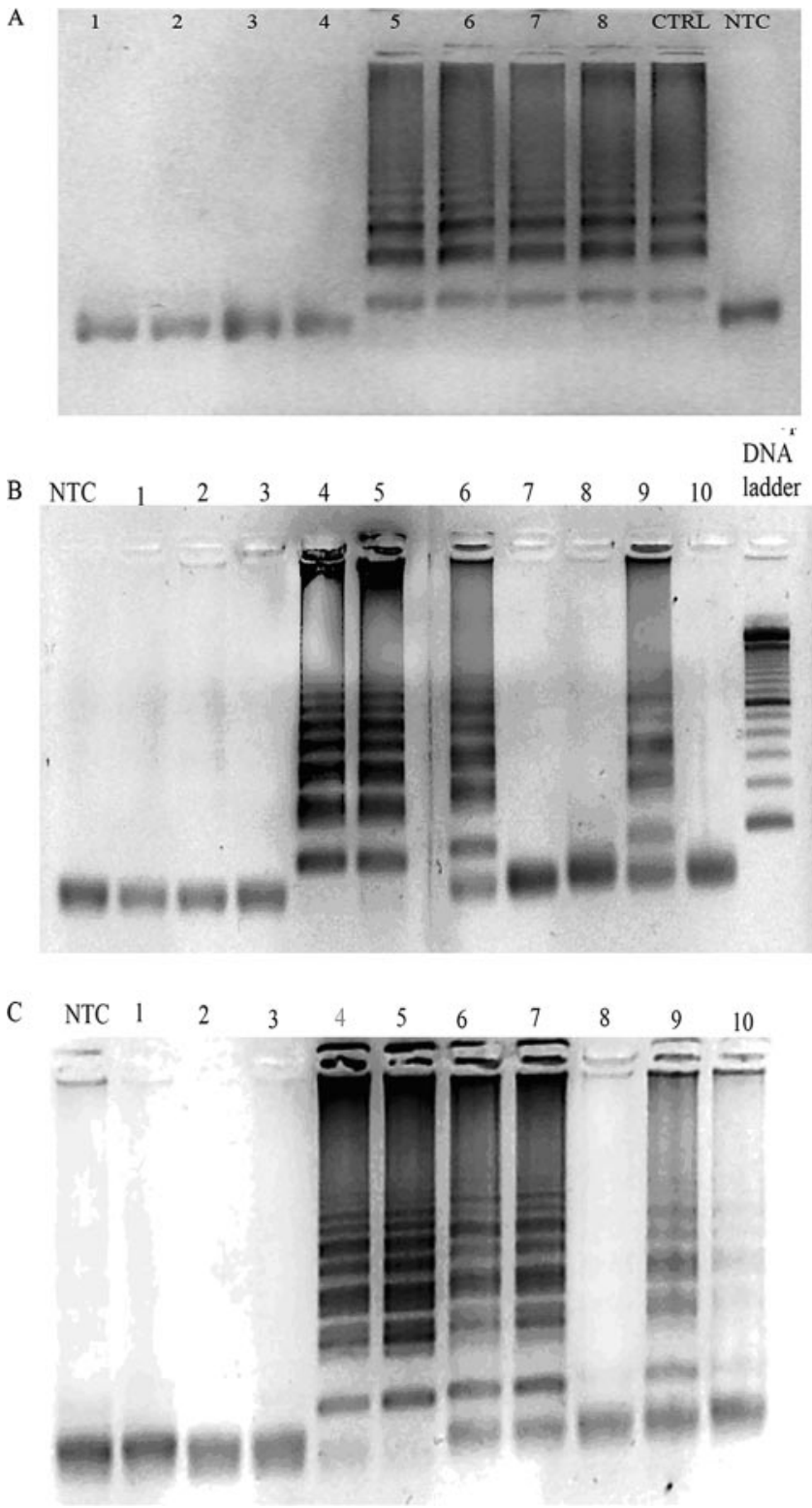

FIG. 4. BKV LAMP with plasma and urine samples from renal transplant patients. (A) Four plasma samples from a single patient with different viral loads assayed with and without heating of samples. The LAMP reaction was negative in unprocessed and unheated plasma samples (lanes 1 to 4 ) and positive in the plasma only after heat treatment of the same samples for 10 min (lanes 5 to 8 ) or after DNA extraction (CTRL, lane 9). Lane 1, 2,264 copies/ml; lane 2, 333,200 copies/ml; lane 3, 50,480 copies/ml; lane 4, 157,200 copies/ml. Lanes 5 to 8 show the same four samples after heat treatment. Lane 5, 2,264 copies $/ \mathrm{ml}$ (heated plasma); lane 6, 333,200 copies/ml (heated plasma); lane 7, 50,480 copies/ml (heated plasma); lane 8, 157,200 copies/ml (heated plasma); lane 9, positive control with extracted DNA (333,200 copies/ml). (B) The LAMP reaction was carried out with unextracted, freshly voided urine samples from 10 renal transplant patients. The corresponding BKV DNA copies were as follows: lane 1, 58,000 copies $/ \mathrm{ml}$; lane 2, 6,000 copies $/ \mathrm{ml}$; lane 3, 40,000 copies $/ \mathrm{ml}$; lane 4, 3.4E + 9 copies/ $\mathrm{ml}$; lane 5,1.7E+9 copies/ml; lane 6, 54,000 copies/ml; lane 7, 110,000 copies $/ \mathrm{ml}$; lane 8, 390,000 copies/ml; lane 9, 52,000 copies/ml; lane 10, 20,000 copies $/ \mathrm{ml}$. (C) The BKV LAMP reaction was carried out with DNA extracted from the same urine samples as those shown in panel B. Note that the reaction was now strongly positive in lane 7 and faintly positive in lane 10 , in addition to lanes $4,5,6$, and 9 in panel $\mathrm{B}$. the clinical utility of PCR-based techniques, they have the inherent disadvantage of being time-consuming as well as requiring considerable operator skill and expensive equipment. We demonstrate that the LAMP technique can be used to amplify BKV DNA under isothermal conditions with high specificity and efficiency. It has a minimal equipment requirement and can be accomplished in 1 hour or less. Moreover, we have also shown that the LAMP reaction can be performed even with unprocessed urine samples and minimally processed (i.e., heat-treated) plasma samples without the need for DNA extraction. Thus, this technique can potentially be developed to screen for virus in "point of care" settings, such as outpatient clinics.

The LAMP method is based on autocycling strand displacement DNA synthesis performed by the Bst DNA polymerase enzyme and was first described by Notomi et al. (14). The principle underlying LAMP is complex, and excellent details of the technique are available in two recent publications $(12,14)$. Briefly, the LAMP assay requires isothermal strand displacement and the amplicons are stem-loop DNA structures with several inverted repeats of the target. The amplicons are formed after multiple rounds of DNA amplification and have structures with multiple loops. Typically, LAMP utilizes four specifically designed primers (two pairs of outer and inner primers) and isothermal Bst DNA polymerase, which has strand displacement activity. Nagamine et al. reported a further improvement by introducing another set of primers, called loop primers, in the reaction (12). Loop primers hybridize to the stem-loops that are formed in the initial phases of LAMP and act by providing additional priming for the DNA polymerase for strand displacement (12). The use of these additional two primers (a total of six primers) further improves the amplification efficiency of the reaction, as originally reported by Nagamine et al. and also confirmed in our studies. The amplicons are typically seen as multiple bands on agarose gel electrophoresis, in contrast to the single band seen with PCR.

An important advantage of LAMP is that the thermal-cycling needs of the PCR method are completely avoided (12). Also, the LAMP assay produces a large amount of amplified product, resulting in easier detection by visual inspection, by observation of either an increase in turbidity caused by generation of magnesium pyrophosphate or a color change after addition of SYBR green I dye $(10,11,12,14)$. The change in turbidity can also be measured quantitatively and in real-time mode by using a real-time turbidimeter $(8,9,15)$. However, the increase in turbidity can be difficult to appreciate and we found it difficult to detect a change in turbidity visually in our experiments. However, visual detection of a red-to-green color change with SYBR green I was easily accomplished and always correlated with gel electrophoresis findings. Our ability to visually detect a positive reaction was significantly improved by using UV transilluminators, including a portable blue light transilluminator that recently became available and can be useful in developing the assay for "point-of-care applications" settings (Fig. 3C). One relative limitation of the LAMP assay is that the color change appears at a visual level to be an all-ornone phenomenon (i.e., a "yes" or a "no") and did not behave in a semiquantitative manner, as the various shades of green for different viral loads were not easily visually distinguishable. Although we did not intend to design a quantitative or semi- 
quantitative LAMP assay, the time required to develop a color change with SYBR green can possibly be used to get a semiquantitative idea of the viral load. The color change occurs earlier at higher viral loads (i.e., $<30 \mathrm{~min}$ incubation for viral loads of $>10 \mathrm{E} 7$ copies) and takes longer for lower loads (60 min for 10,000 to 100,000 copies/ml).

The LAMP assay is more complex to design than PCR assays. However, once developed, LAMP is much easier to perform than PCR or real-time PCR and requires a simple addition of unprocessed urine or plasma to a tube containing a mixture of primers, enzyme, and buffers, followed by warming for 30 to $60 \mathrm{~min}$ at 60 to $65^{\circ} \mathrm{C}$. Hence, the hands-on time of approximately $1 \mathrm{~h}$ or less for LAMP can be comparable to or even significantly shorter than that required for PCR or realtime PCR. Another advantage of LAMP is that, in contrast to $\mathrm{PCR}$, it requires no DNA extraction, which can be a major obstacle in widespread employment of DNA amplification assays. Our LAMP assay performed successfully without DNA extraction for both urine and plasma samples. Unprocessed, freshly voided urine is considered to contain PCR inhibitors but was successfully tested with BKV LAMP assays. Although there was a loss of some sensitivity with the use of unprocessed samples (versus what was found for extracted DNA samples), the assay could still be very useful in clinical situations. The reasons for the variable performance of the assay for viral loads of less than 100,000 copies/ml of urine are not clear but may be multifactorial. One possibility is that the LAMP assay demands more integrity of the target, owing to the many primers that need to be annealed to the different regions, than PCR. Since the LAMP assays were tested on stored samples, it is possible that there may have been partial degradation of the target DNA in the region of LAMP primers but not in the region that is amplified by PCR primers. Another possibility is the presence of urine-specific inhibitors of LAMP reaction. The patients with false-negative urine LAMP results did not have any obvious clinical differences from other patients and also did not have any proteinuria, which can possibly act as an inhibitor. However, various drugs, metabolites, chemicals, and dietary agents are excreted in urine, and some of these may affect urinary LAMP more than plasma LAMP assays. Further refinements of technique are clearly needed to improve the sensitivity and the semiquantitative aspects of the LAMP assays as well as to address the issues related to as-yet-unrecognized urinary "inhibitors." Also, additional studies, including validation of visual testing with a larger number of clinical samples and proficiency testing, will be required before these assays can be employed in routine clinical laboratories.

BKVAN is emerging as an important cause of renal allograft dysfunction and can resemble acute rejection (AR) both clinically and histologically $(3,6,7,13)$. Differentiation between BKVAN and AR is important, however, because the treatments for the two conditions are diametrically opposite. Several studies have shown that BK viruria is a requirement for development of BKVAN and generally precedes the appearance of viremia and full-blown nephropathy by several weeks to months. BKV DNA detection in urine by PCR is also much more sensitive than viral detection by urinary cytology (decoy cells) and also viral detection by immunohistochemistry or in situ hybridization on allograft biopsy $(2,3,16)$. However, asymptomatic BK viruria can be seen in up to $30 \%$ of kidney transplant patients and only approximately 6 to $10 \%$ of these patients develop full-blown BKVAN $(13,19)$. The presence of a high viral load is now considered to be more correlated with risk for development of BKVAN than just the presence of virus in urine $(16,17)$. Our previous studies have shown that viral loads of $>10 \mathrm{E} 7$ copies $/ \mathrm{ml}$ in urine and $>10,000$ copies $/ \mathrm{ml}$ in blood are reasonable threshold loads above which a renal transplant patient is more likely to have or develop BKVAN $(16,17,19)$. BKV LAMP showed consistent performance in our studies at urine loads of $\geq 10 \mathrm{E} 5$ copies $/ \mathrm{ml}$ and for $>2,000$ copies/ml of plasma even when performed with unextracted samples. These detection limits are well below the viral loads that are considered to be clinically significant. Hence, this assay could easily be used in point-of-care settings to detect high-risk patients with viral loads above the critical levels. Demonstration of BKV in urine and blood can alert a clinician to the possibility of BKVAN in a patient presenting an asymptomatic rise in serum creatinine, which is most often attributed to AR in renal transplant recipients.

In summary, we report our early experience with the development and performance characteristics of a BKV LAMP assay. It is a novel technique that can possibly be used for rapid diagnosis of BKV infection not only in laboratories but also in an outpatient clinic setting. A reduction in immunosuppression is now considered the first-line therapy for BKV infections in renal transplant recipients $(1,4,7)$. However, physicians currently depend on PCR results for BKV detection, which might take 2 to 3 days or longer to arrive in many clinic settings. Thus, the LAMP assay for BKV can potentially be used to guide therapeutic decisions in outpatient clinics itself, especially if a urine sample becomes positive after a short, 30-min incubation and is accompanied by a plasma sample that shows positivity, suggesting that the patient is more likely to have a clinically significant viral load. Further large-scale studies for determination of the sensitivity, specificity, and clinical utility of this new method will be needed before this method can find wider clinical applicability.

\section{ACKNOWLEDGMENT}

This work was supported by an NIH award (R01 AI060602) to A.V.

\section{REFERENCES}

1. Brennan, D. C., I. Agha, D. L. Bohl, M. A. Schnitzler, K. L. Hardinger, M. Lockwood, et al. 2005. Incidence of BK with tacrolimus versus cyclosporine and impact of preemptive immunosuppression reduction. Am. J. Transplant. 5:582-594.

2. Drachenberg, C. B., J. C. Papadimitriou, H. H. Hirsch, R. Wali, C. Crowder, J. Nogueira, et al. 2004. Histological patterns of polyomavirus nephropathy: correlation with graft outcome and viral load. Am. J. Transplant. 4:20822092.

3. Drachenberg, C. B., H. H. Hirsch, E. Ramos, and J. C. Papadimitriou. 2005. Polyomavirus disease in renal transplantation: review of pathological findings and diagnostic methods. Hum. Pathol. 36:1245-1255.

4. Hariharan, S. 2006. BK virus nephritis after renal transplantation. Kidney Int. 69:655-662.

5. Hirsch, H. H. 2005. BK virus: opportunity makes a pathogen. Clin. Infect. Dis. 41:354-360.

6. Hirsch, H. H., and J. Steiger. 2003. Polyomavirus BK. Lancet Infect. Dis. 3:611-623.

7. Hirsch, H. H., D. C. Brennan, C. B. Drachenberg, F. Ginevri, J. Gordon, A. P. Limaye, et al. 2005. Polyomavirus-associated nephropathy in renal transplantation: interdisciplinary analyses and recommendations. Transplantation 79:1277-1286.

8. Hong, T. C., Q. L. Mai, D. V. Cuong, M. Parida, H. Minekawa, T. Notomi, et al. 2004. Development and evaluation of a novel loop-mediated isothermal amplification method for rapid detection of severe acute respiratory syndrome coronavirus. J. Clin. Microbiol. 42:1956-1961. 
9. Ihira, M. T. Yoshikawa, Y. Enomoto, S. Akimoto, M. Ohashi, S. Suga, et al. 2004. Rapid diagnosis of human herpesvirus 6 infection by a novel DNA amplification method, loop-mediated isothermal amplification. J. Clin. Microbiol. 42:140-145.

10. Mori, Y., K. Nagamine, N. Tomita, and T. Notomi. 2001. Detection o loop-mediated isothermal amplification reaction by turbidity derived from magnesium pyrophosphate formation. Biochem. Biophys. Res. Commun. 289:150-154.

11. Mori, Y., M. Kitao, N. Tomita, and T. Notomi. 2004. Real-time turbidimetry of LAMP reaction for quantifying template DNA. J. Biochem. Biophys. Methods 59:145-157.

12. Nagamine, K., T. Hase, and T. Notomi. 2002. Accelerated reaction by loopmediated isothermal amplification using loop primers. Mol. Cell. Probes 16:223-229.

13. Nickeleit, V., and M. J. Mihatsch. 2004. Polyomavirus allograft nephropathy and concurrent acute rejection: a diagnostic and therapeutic challenge Am. J. Transplant. 4:838-839.

14. Notomi, T., H. Okayama, H. Masubuchi, T. Yonekawa, K. Watanabe, N. Amino, et al. 2000. Loop-mediated isothermal amplification of DNA Nucleic Acids Res. 2:E63.

15. Poon, L. L., B. W. Wong, E. H. Ma, K. H. Chan, L. M. Chow, W. Abeyewickreme, N. Tangpukdee, K. Y. Yuen, Y. Guan, S. Looareesuwan, and J. S. Peiris. 2006.
Sensitive and inexpensive molecular test for falciparum malaria: detecting Plasmodium falciparum DNA directly from heat-treated blood by loop-mediated isothermal amplification. Clin. Chem. 52:303-306.

16. Randhawa, P., A. Ho, R. Shapiro, A. Vats, P. Swalsky, S. Finkelstein, et al. 2004. Correlates of quantitative measurement of BK polyomavirus (BKV) DNA with clinical course of BKV infection in renal transplant patients. J. Clin. Microbiol. 42:1176-1180.

17. Randhawa, P., R. Shapiro, and A. Vats. 2005. Quantitation of DNA of polyomaviruses BK and JC in human kidneys. J. Infect. Dis. 192:504-509.

18. Suzuki, R., T. Yoshikawa, M. Ihira, Y. Enomoto, S. Inagaki, K. Matsumoto, et al. 2006. Development of the loop-mediated isothermal amplification method for rapid detection of cytomegalovirus DNA. J. Virol. Methods 132:216-221.

19. Vats, A., R. Shapiro, P. Singh Randhawa, V. Scantlebury, A. Tuzuner, M. Saxena, M. L. Moritz, T. J. Beattie, T. Gonwa, M. D. Green, and D. Ellis 2003. Quantitative viral load monitoring and cidofovir therapy for the management of BK virus-associated nephropathy in children and adults. Transplantation 75:105-112.

20. Yoshikawa, T., M. Ihira, S. Akimoto, C. Usui, F. Miyake, S. Suga, et al. 2004 Detection of human herpesvirus 7 DNA by loop-mediated isothermal amplification. J. Clin. Microbiol. 42:1348-1352. 